\title{
Direct visualization of carbon dioxide field flooding: Optical and concentration level comparison of diffusor effectiveness
}

\author{
Stijn Vandenberghe, PhD, David Iseli, BSc, and Stefanos Demertzis, MD
}

\section{ABSTRACT}

Objective: Carbon dioxide field flooding during open-heart surgery is intended to avoid blood-air contact, bubble formation, and embolism, and therefore potential neurologic and other ischemic complications. The inert gas is invisible, and thus its use and effectiveness are heavily debated. We intended to provide better insight in the behavior of the gas via direct concentration measurements and visualization of the gas cloud.

Methods: A transparent rectangular model of the open thorax was created, foreseen with carbon dioxide concentration sensors in 2 locations (atrial and aortic incisions), and placed in an optical test bench that amplifies the diffraction gradients. Six different commonly used carbon dioxide diffusors (3 commercial, 3 improvised) were tested with different flow rates of gas delivery $(1,4,7,10$ standard liter per minute [SLPM]) and combined with the application of suction.

Results: The imaging reveals that commercially available diffusors generally create less turbulent flow than improvised diffusors, which is supported by the concentration measurements where improvised diffusors cannot generate a $100 \%$ carbon dioxide atmosphere at the aorta incision location. The atrial incision is easier to protect: $0 \%$ air with all commercial devices for all flow rates greater than 1 SLPM. A flow rate of 1 SLPM does not create an inert atmosphere with any device.

Conclusions: The optically observed carbon dioxide atmosphere is unstable and influenced by many factors. The device used for diffusion and the flow rate are important determinants of the maximum gas concentration that can be achieved, as is the location where this is measured. (J Thorac Cardiovasc Surg 2020;159:958-68)

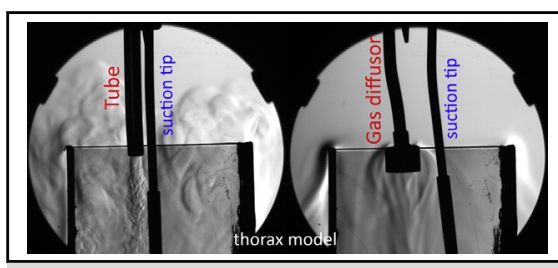

$\mathrm{CO}_{2}$ flow at $10 \mathrm{SLPM}$ from an improvised delivery tube (L) versus a commercial diffusor $(\mathrm{R})$.

\section{Central Message}

$\mathrm{CO}_{2}$ field flooding is not well understood. We provided insight in diffusor effectiveness via visualization and concentration measurements and demonstrated an unstable $\mathrm{CO}_{2}$ atmosphere.

\section{Perspective}

Many misunderstandings exist about $\mathrm{CO}_{2}$ field flooding, mainly because the gas cannot be observed. This engineering study literally reveals the gas behavior, and the observations are corroborated by instantaneous concentration measurements. The reported findings should be mostly of interest to cardiac surgeons, who can easily improve cerebral protection of their next patient.

See Commentaries on pages 969 and 970.
Blood circulation is a closed system shielded from the extracorporeal environment. During open or major vascular surgery, atmospheric air can freely enter and form air bubbles in the blood, which after removal of the clamps or recommencing circulation may be driven toward the brain and cause neurologic damage. ${ }^{1}$ This is caused not only by the blockage of an artery itself (even a $2.5-\mu \mathrm{L}$ bubble can

From the Department of Cardiac Surgery, Cardiocentro Ticino, Lugano, Switzerland. This study was in part supported by a grant from the Swiss Commission for Technology and Innovation (CTI 19212.1 PFLS-LS) and in part by institutional funding.

Received for publication Nov 29, 2018; revisions received April 5, 2019; accepted for publication April 9, 2019; available ahead of print June 11, 2019.

Address for reprints: Stijn Vandenberghe, PhD, Cardiocentro Ticino, Via ai Soï 24 6807, Taverne-Toricella, Switzerland (E-mail: Stijn.vandenberghe@cardiocentro. org).

0022-5223/\$36.00

Copyright (c) 2019 by The American Association for Thoracic Surgery

https://doi.org/10.1016/j.jtcvs.2019.04.040 disrupt brain function in less than 30 seconds $^{2}$ ) but also by the an inflammatory response. In addition to stroke as the most feared outcome, another complication that may be caused by air embolism is silent infarction and its potential for postoperative cognitive disorders, ${ }^{3-5}$ for which no clear diagnostic and measurable outcomes are available.

Therefore, it is an important part of a cardiac surgery procedure to carefully remove air bubbles from the blood before liberating heart and vessels (deairing). The

Scanning this $\mathrm{QR}$ code will take you to the article title page to access supplementary information.

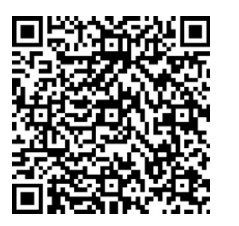




\section{Abbreviations and Acronyms}

$\mathrm{CO}_{2}=$ carbon dioxide

$\mathrm{IQR}=$ interquartile range

SLPM $=$ standard liter per minute

traditional surgical methods, such as vent catheters, aspirating with needles, shaking the heart, or tilting the operating table during pulmonary expansion, only remove a portion of the entrapped air, as is repeatedly proven with intraoperative transesophageal echography. 6,

The approach to create an inert atmosphere in the thorax with carbon dioxide $\left(\mathrm{CO}_{2}\right)$ appears to be more safe and consistent, but has been a source of controversy ever since its inception in the late 1950s. ${ }^{8}$ The concept is well known: because $\mathrm{CO}_{2}$ is approximately $70 \%$ heavier (density at standard temperature and pressure: $1.98 \mathrm{~kg} / \mathrm{m}^{3}$ ) than air, the gas will fill the thorax cavity and push the air up and away from the blood. $\mathrm{CO}_{2}$ easily dissolves in blood (solubility is $25 \times$ that of air) and does not have the risk of blocking a vessel long enough to cause ischemic damage. But $\mathrm{CO}_{2}$ is also an invisible gas, and thus it cannot be observed if it effectively fills the thorax, which quantities need to be applied, how long it stays present in the thorax, and how dangerous it could be for the surgical staff.

Despite the long history of $\mathrm{CO}_{2}$ field flooding, relatively few scientific studies (barely $300^{9,10}$ ) have been performed, with inconclusive results, ${ }^{11,12}$ and the ongoing debate about the effectiveness causes a divide between proponents ${ }^{13-16}$ and opponents ${ }^{17,18}$ of this method. Surgeons who are convinced that $\mathrm{CO}_{2}$ forms a protective atmosphere to prevent or reduce blood-air contact may still use it in an ineffective way because of the lack of understanding of the gas behavior. The resulting inconsistent application of $\mathrm{CO}_{2}$ makes it difficult to prove or disprove the effectiveness of $\mathrm{CO}_{2}$ field flooding in clinical trials.

The last 15 years have seen a renewed interest in assessing the effectiveness of $\mathrm{CO}_{2}$ field flooding, due in part to improved ultrasound technology to assess the embolic load. $^{6,7,19}$ With the development of the first commercial $\mathrm{CO}_{2}$ diffusor, a range of laboratory studies were performed that demonstrated that flow rate, presence of

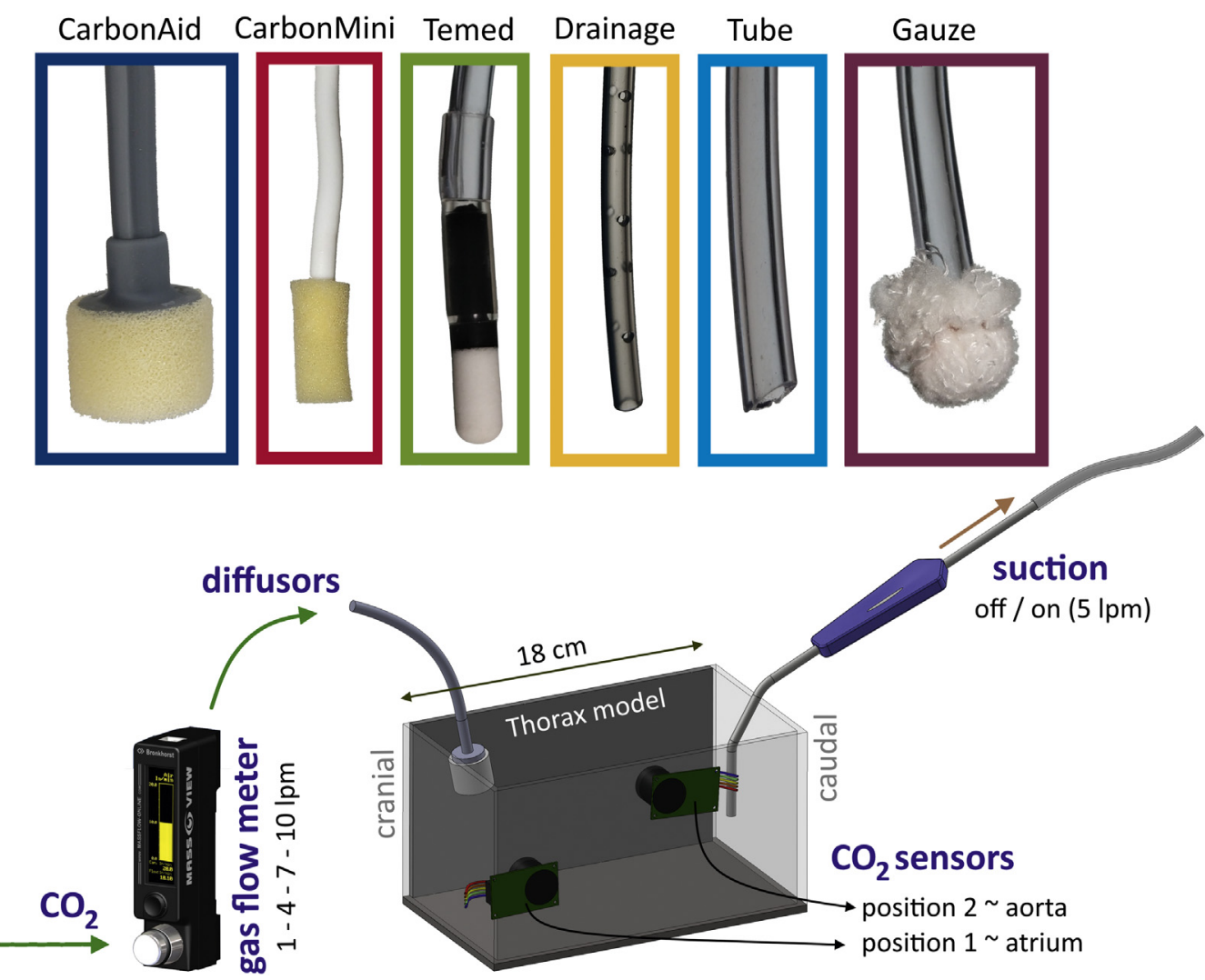

FIGURE 1. Top: Color-coded photographs of the 6 different diffusors that were tested in the thorax model. Bottom: sketch of the test setup consisting of a box representing the open thorax and indicating the locations of the diffusor under test, the suction tip, and the high-speed $\mathrm{CO}_{2}$ concentration sensors. The green arrows indicate the flow of $\mathrm{CO}_{2}$ gas into the model, and the brown arrow indicates the gas evacuation via the suction tip that was used in parts of the protocol (Table 1). $\mathrm{CO}_{2}$, Carbon dioxide. 
suction, and design of the diffusor make a notable difference in the remaining air content in the thorax. ${ }^{20}$

In this article, we aim to contribute to the better understanding of $\mathrm{CO}_{2}$ field flooding via direct visualization of the gas mixing, combined with high-speed measurements of the $\mathrm{CO}_{2}$ concentration directly inside a thorax model.

\section{MATERIAL AND METHODS \\ Setup}

A simplified model of the open thorax was created as a rectangular box with $18-\mathrm{cm}$ length and $10-\mathrm{cm}$ width and height and airtight seals (Figure 1). Two opposing panels were made from 2-mm thick acrylic to enable visualization as a long-axis projection. In 1 of the side panels, 2 high-speed, wide range $(0 \%-100 \%) \mathrm{CO}_{2}$ sensors were installed (Gas Sensing Solutions Ltd, Cumbernald, UK): a Cozir $(2 \mathrm{~Hz}$ ) in cranial mid-height position (simulating the position of the left atrium) and a SprintIR $(20 \mathrm{~Hz})$ in a central anterior (top-height) position (simulating the anatomic position of the ascending aorta). The sensors were calibrated before each measurement set with medical grade $\mathrm{CO}_{2}$ gas.

Six different $\mathrm{CO}_{2}$ diffusors were subsequently installed in the most cranial position of the model and connected to $\mathrm{a}_{2}$ tank via a regulator and digital gas flow meter (Massview, Bronkhorst High-Tech b.v., Ruurlo, The Netherlands). The flow meter was calibrated for $\mathrm{CO}_{2}$ in standard liter per minute (SLPM); a volumetric flow rate scaled to standard temperature and pressure $\left(0^{\circ} \mathrm{C}, 1 \mathrm{bar}\right)$, which enables comparison of gas flows under pressure.

The model and instrumentation were placed in a Z-type Schlieren setup with 2 150-mm spherical mirrors, with the following components all installed on XYZ-micrometer stages for precise alignment and focusing: a point light source, a knife blade, and a CCD camera (AC1920, Basler AG, Ahrensburg, Germany). The Schlieren technique amplifies the small diffraction gradient between $\mathrm{CO}_{2}$ and air. Figure 2 shows that with the naked eye, no gas can be observed coming out of the diffusor, while simultaneously the video camera (see TV monitor) captures the Schlieren effect and clearly shows the $\mathrm{CO}_{2}$ gas flow. Isolated images of the Schlieren effect are displayed in Figure 3. The boundary between $\mathrm{CO}_{2}$ gas and air is indicated by shadows that cast a 3-dimensional effect.

\section{Diffusors}

Three commercially available $\mathrm{CO}_{2}$ diffusors were tested:

- CarbonAid (CardiaInnovation AB, Kungens Kurva, Sweden)

- CarbonMini (CardiaInnovation AB)

- Temed Gas Diffusor (Temed, Sidlesham, United Kingdom).

These were compared with 3 improvisations that are frequently seen in cardiac surgery:

- multi-perforated drainage catheter (Drenofast; Iberhospitex SA, Barcelona, Spain),

- a $1 / 8^{\prime \prime} \times 1 \times 16^{\prime \prime}$ polyvinyl chloride tube

- a $1 / 8^{\prime \prime} \times 1 / 16^{\prime \prime}$ polyvinyl chloride tube covered with 3 layers of gauze.

These are further referred to as "drainage," "tube," and "gauze," respectively.

\section{Protocol}

Video data were recorded with the optical system while $\mathrm{CO}_{2}$ concentration data were digitally acquired from the $\mathrm{CO}_{2}$ sensors. An overview of the performed measurements, split in experiments $\mathrm{A}$ and $\mathrm{B}$, is presented in Table 1 . Table 1 was replicated 3 times at different dates, and in between, the setup was completely rebuilt. For experiment A, all 6 diffusors were sequentially tested while aligned with the vertical axis, and at 4 different

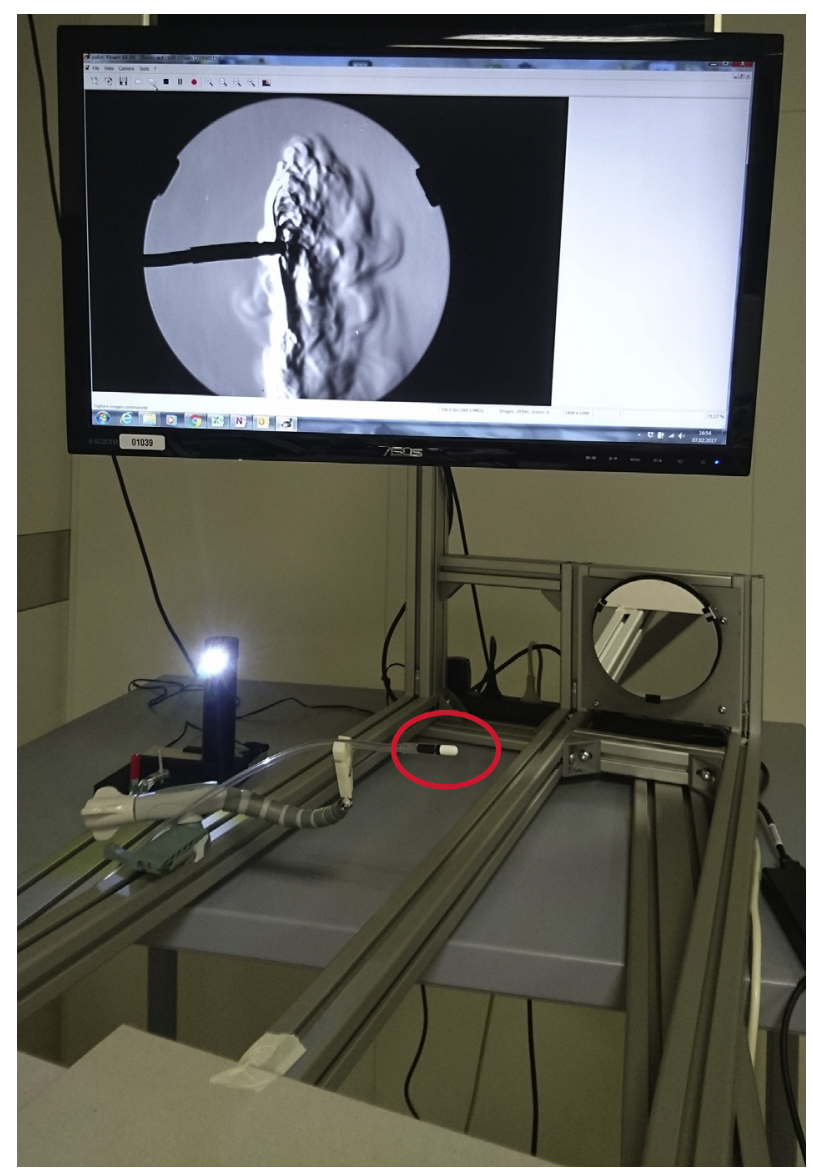

FIGURE 2. Illustration of the suitability of the Schlieren principle for the study of $\mathrm{CO}_{2}$ field-flooding: $\mathrm{CO}_{2}$ gas coming from a gas diffusor at 4 SLPM cannot be observed with the naked eye (red circle, direct photographer's view), while simultaneously an obvious gas cloud can be seen on the LCD screen, which comes from a live-view camera aiming at the setup's spherical mirrors.

flow rates $(1,4,7$, or 10 SLPM), without the use of suction. In experiment $\mathrm{B}$, only the 3 commercial diffusors were tested under different conditions: a second data set with the diffusor tilted $45^{\circ}$ in the medial plane and a third data set in the standard vertical orientation but combined with the application of suction. For this, a suction tip was installed vertically at the caudal side of the model, and a regulated suction flow of $5 \mathrm{~L} / \mathrm{min}$ was imposed.

Before each measurement, the $\mathrm{CO}_{2}$ tank was opened, and the regulator modified to read the desired gas flow $\left(1,4,7\right.$, or 10 SLPM). The $\mathrm{CO}_{2}$ delivery tube was then clamped, and the thorax model emptied via suction until both sensors indicated less than $1 \% \mathrm{CO}_{2}$ concentration. Recording was started, and the clamp was removed to immediately deliver the $\mathrm{CO}_{2}$ gas at the desired flow rate. This was maintained until the $\mathrm{CO}_{2}$ concentration reached a plateau.

\section{Data Processing}

A custom algorithm written in MATLAB software (MathWorks Inc, Natick, Mass) detected the concentration plateau from each concentration measurement file. Two continuous quantitative variables were extracted from the plateau (Figure 4): the maximum $\mathrm{CO}_{2}$ concentration that was achieved (ie, the average of the plateau) and the time to reach the plateau (rise time). The data are graphically presented as Tukey box plots and reported in the text as median and interquartile range (IQR). STATA software 


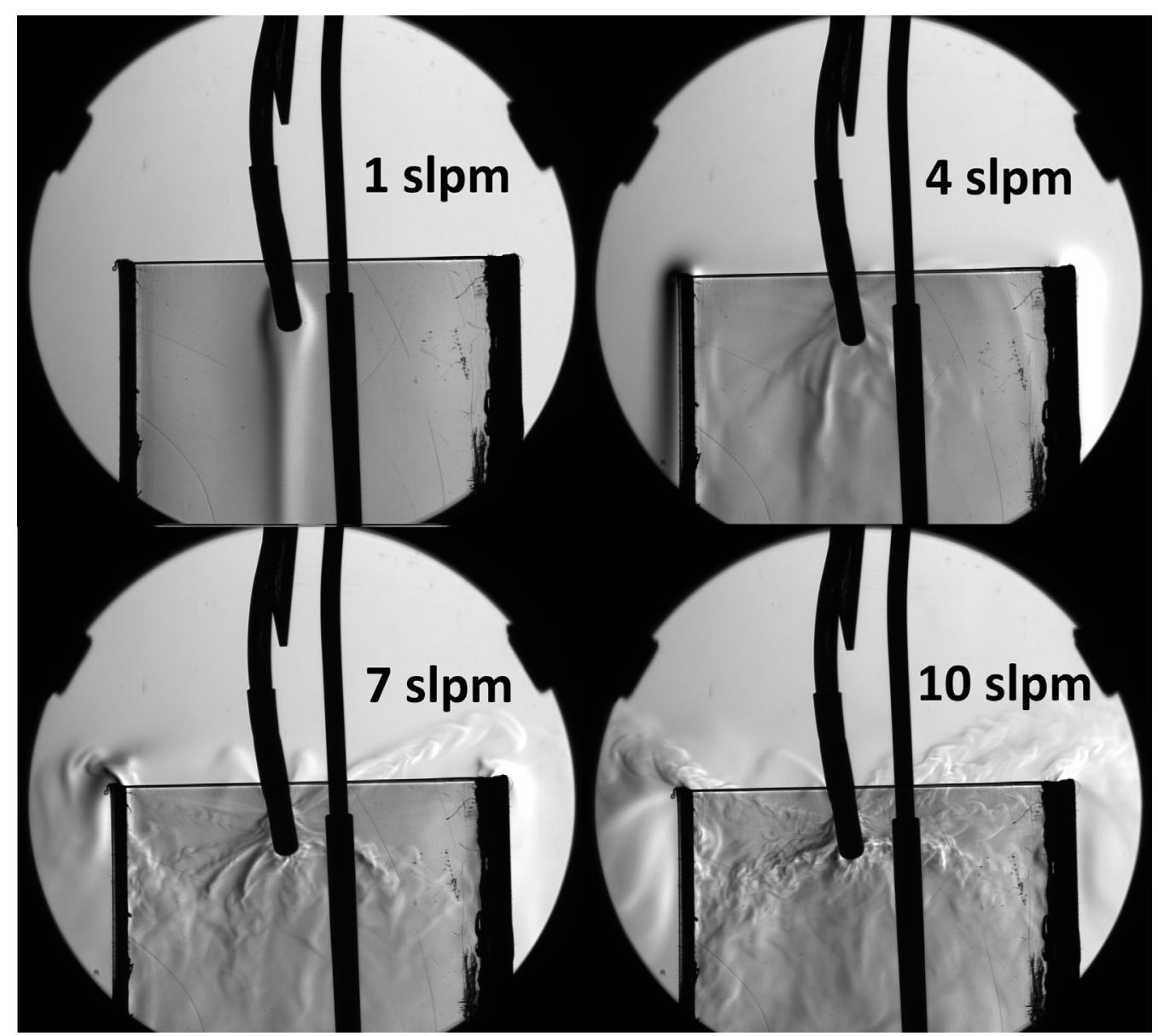

FIGURE 3. Demonstration of gas clouds generated by a Temed $\mathrm{CO}_{2}$ diffusor at different flow rates $(1,4,7$, or 10 SLPM). The black vertical rod on the right side of the diffusor is the shadow of the caudally placed suction tip, which was not sucking during these recordings. SLPM, Standard liter per minute.

(StataCorp LLC, College Station, Tex) was used to fit multi-level, mixedeffects models to the data of each experiment, with the covariate "device" defined as random effect. Fixed factors were "position" and "flow rate" (experiment A), and "suction" for experiment B (tilting was excluded after an initial screening). A Bonferroni correction of the $P$ levels was applied for subsequent pairwise comparisons.

TABLE 1. Overview of the test protocol

\begin{tabular}{lllll}
\hline \multicolumn{1}{c}{ Diffusor } & & \multicolumn{4}{c}{ Experiment A } & & Condition & No. of runs & Replications \\
\hline CarbonAid & Commercial & $1 / 4 / 7 / 10$ SLPM & Vertical & 3 \\
CarbonMini & & & & \\
Temed & & & & \\
Drainage catheter & Improvised & & & \\
Tube & & & & \\
Tube + gauze & & & & \\
\end{tabular}

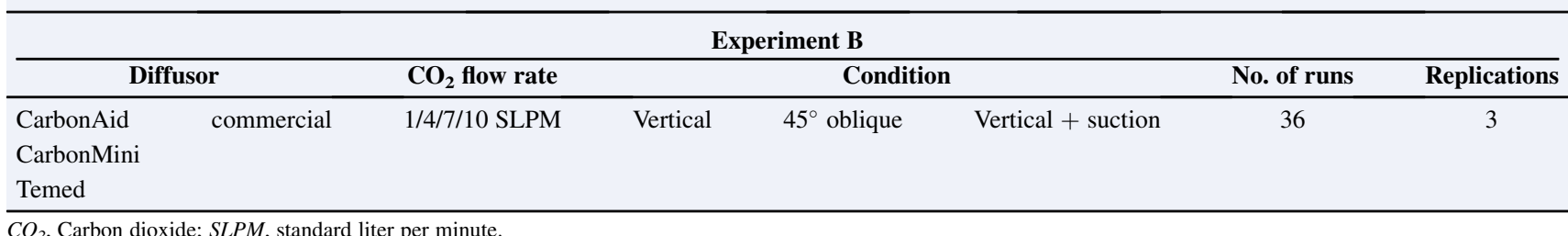

$\mathrm{CO}_{2}$, Carbon dioxide; SLPM, standard liter per minute. 

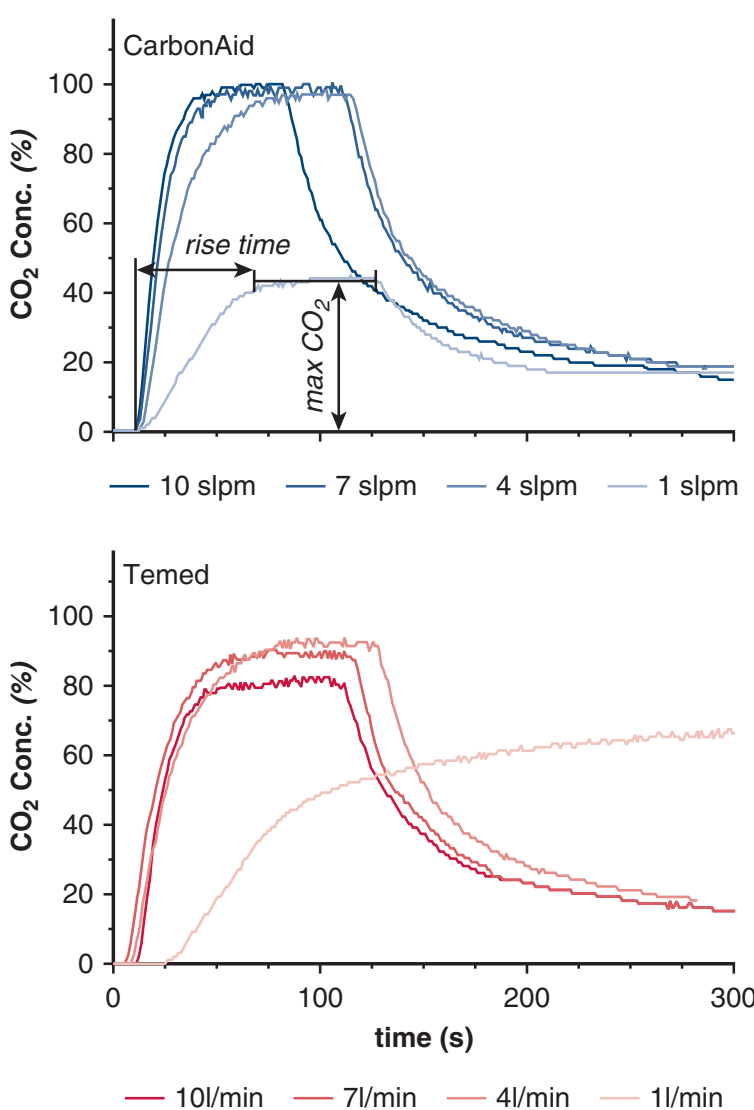

FIGURE 4. Comparison of 2 commercial gas diffusors and the effect of gas delivery flow rates $(1,4,7$, or 10 SLPM) on the maximum achievable gas concentration in the thorax and the rise time needed to achieve this. The right side of each graph (>100 seconds) illustrates how rapidly gas concentration decays when delivery is cut off. These data were obtained from a $\mathrm{CO}_{2}$ concentration sensor in position 2 in the thorax model (simulation of aorta incision) and without application of suction from a blood sucker. $\mathrm{CO}_{2}$, Carbon dioxide; $S L P M$, standard liter per minute.

\section{RESULTS \\ Experiment A}

The optical results for experiment A, obtained with the Schlieren system, are presented in Videos 1 to 4. Each video concentrates on a specific flow rate and shows parallel frames with the 6 tested devices in the thorax model. The shadow of the diffusor and the suction tip are visible in the frames, although suction was not active during these specific recordings. At the start of gas flow, each video shows outspoken gas clouds, where some devices show high turbulent mixing of $\mathrm{CO}_{2}$ and air, while the CarbonAid device generates a distinctive laminar downflow. Especially with this device, as the thorax keeps filling, the boundary between the gases fades, indicating that the model is completely filled with $\mathrm{CO}_{2}$. While Videos 1 to 4 enable comparison of devices at a single flow rate, Figure 3 shows the comparison of different flow rates for 1 device, namely, the Temed diffusor. It also shows that for this device, higher flow rates generate more drastic turbulent mixing.

When comparing Video 1 (at 1 SLPM) with Videos 2 to 5, or likewise comparing the different panels of Figure 3, it can be observed that at higher flow rates the $\mathrm{CO}_{2}$ gas flows over the edge of the thorax model, but at 1 SLPM it does not. Also the time-varying $\mathrm{CO}_{2}$ concentration depicted in Figure 4 for the Temed and CarbonAid diffusors reveals a big discrepancy between a 1 SLPM flow rate and the other flow rates. The maximum achievable concentration of $\mathrm{CO}_{2}$ in the thorax model is well below $100 \%$ at the aortic incision level if 1 SLPM is used, and it also takes longer to reach a steady state. This is further confirmed in Figure 5, in which a comparison of the maximum achieved $\mathrm{CO}_{2}$ concentration (top) reveals notably lower concentrations for the 1 SLPM flow rate of CarbonAid, CarbonMini, Temed, and Gauze compared with the higher flow rates. The mixed model $\left(r^{2}: 0.96\right)$ shows a significant effect of device, flow rate, position, and the device*flow rate interaction. The Bonferroni pairwise comparison of all devices further reveals that Drainage and Tube generate significantly lower $\mathrm{CO}_{2}$ concentrations than all other devices over all factors, whereas concentrations present at the aorta incision are also significantly lower than at the atrial position. A pairwise comparison of the different devices is presented in Table 2.

The lower panel of Figure 4 also shows that the maximum $\mathrm{CO}_{2}$ concentration achieved with a Temed diffusor at 10 SLPM is actually lower than at 4 and 7 SLPM, indicating that a higher flow rate does not necessarily lead to a higher concentration. Similar trends can be seen in Figure 5, where the Temed, simple tube, and tube with gauze all show decreasing $\mathrm{CO}_{2}$ concentrations for increasing flow rates. It appears that each device has an optimal flow rate, which is for instance 1 SLPM for the drainage catheter, and 4 SLPM for Temed and Gauze. In general, for all flow rates and both positions, the commercial Temed and the improvised Gauze show a good agreement in performance, and no significant differences were found between these devices. The CarbonAid is the only device that achieves a $100 \% \mathrm{CO}_{2}$ atmosphere for all flow rates (except 1 SLPM) in all positions. At the highest flow rate of 10 SLPM, it is significantly more effective than all other devices. The least effective device at these flow rates is the multi-perforated drainage catheter, which still allows/entrains approximately $80 \%$ of air into the thorax and is thereby significantly worse than all other devices. In Video 3 or the "drainage" section of Figure 6, the drainage catheter shows extraordinary gas delivery in the form of a massive cloud of small turbulences that rises high above the thorax model, while other devices generate less turbulent clouds where the gas gently flows over the model edges.

The rise time is significantly higher at 1 SLPM versus the other flow rates over all factors, whereas there is no 


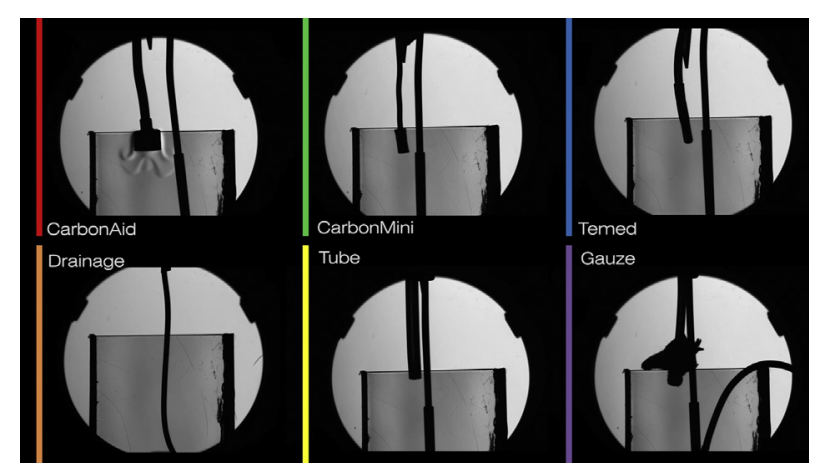

VIDEO 1. All tested diffusors are shown side by side, dispersing $\mathrm{CO}_{2}$ in the rectangular thorax model at a flow rate of 1 SLPM. Video available at: https://www.jtcvs.org/article/S0022-5223(19)30936-5/fulltext.

significant contribution of the position to the fitted mixed effects model ( $\left.{ }^{2}: 0.90\right)$. At 1 SLPM, it takes approximately 3 minutes to fill the thorax with a steady atmosphere of $\mathrm{CO}_{2}$, whereas at other flow rates it takes only 1 minute. It appears that at the highest flow rates $(4,7$, or 10 SLPM), particularly in position 2 , the drainage catheter stands out positively for reaching its steady-state plateau faster than the other devices: a median rise time of 26 seconds (IQR, 14) for the drainage catheter (at 4 SLPM; position 2) versus 71 seconds (IQR, 16) for all the other devices combined. However, it needs to be considered that this catheter has a lower plateau: $16 \% \mathrm{CO}_{2}(\mathrm{IQR}, 7.5)$ versus $94 \%$ (IQR, 6.5) by the other devices for the same condition, and is thus far worse at creating a protective $\mathrm{CO}_{2}$ environment. Overall, every factor introduced in the multilevel model had a statistically significant impact on maximum $\mathrm{CO}_{2}$ concentration and rise time, with the exception of "position," which had no significant effect on rise time. The rise time could be considered an indicator of how quickly the $\mathrm{CO}_{2}$ atmosphere could recover to its original composition after it has been disturbed, for instance by surgical maneuvers.

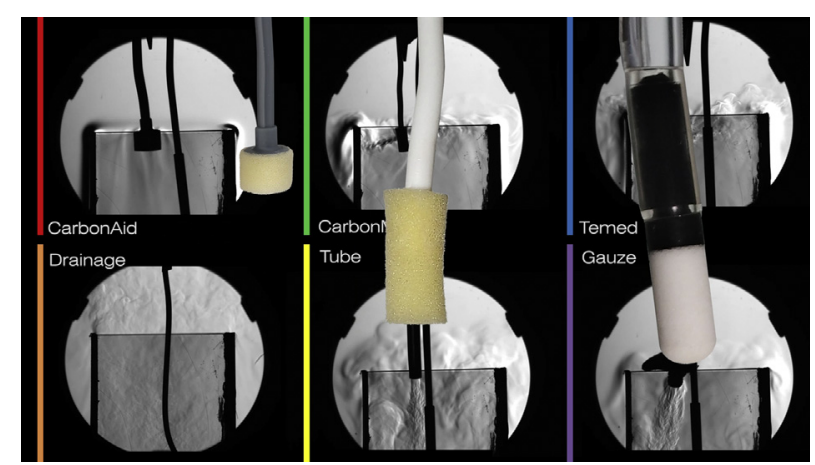

VIDEO 2. All tested diffusors are shown side by side, dispersing $\mathrm{CO}_{2}$ in the rectangular thorax model at a flow rate of 4 SLPM. Video available at: https://www.jtcvs.org/article/S0022-5223(19)30936-5/fulltext.

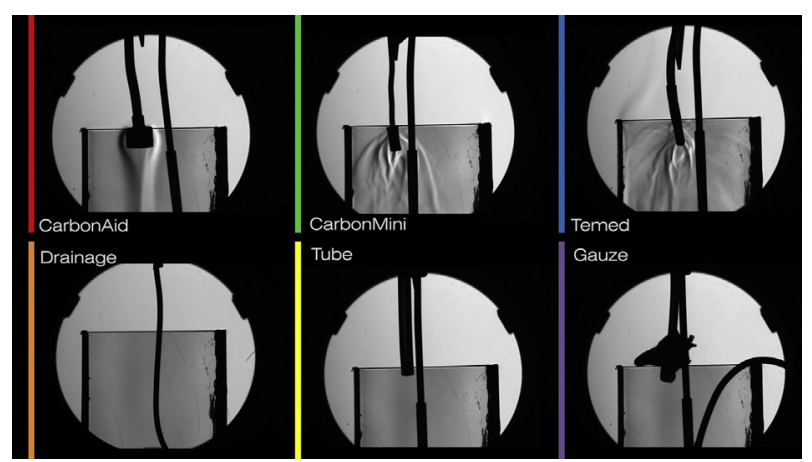

VIDEO 3. All tested diffusors are shown side by side, dispersing $\mathrm{CO}_{2}$ in the rectangular thorax model at a flow rate of 7 SLPM. Video available at: https://www.jtcvs.org/article/S0022-5223(19)30936-5/fulltext.

\section{Experiment B}

Figure 7 shows that a suction of $5 \mathrm{~L} / \mathrm{min}$ has a negative

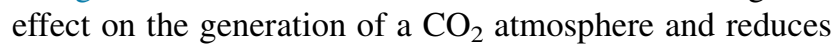
the maximum achievable concentration to the effect that no device at any of the tested flow rates was able to generate a consistent $100 \% \mathrm{CO}_{2}$ reading in the "aorta incision" position. For the "atrium incision" region, only CarbonAid and CarbonMini could achieve a consistent $100 \%$ reading $(\mathrm{IQR}<3)$ with 7 and 10 SLPM flow rates. The mixed effects model $\left(r^{2}: 0.93\right)$ confirms that suction has a significant impact on the maximum $\mathrm{CO}_{2}$ concentration. The pairwise comparison further shows that also with suction, the lowest flow rate performs significantly worse than the other flow rates: $\mathrm{CO}_{2}$ values do not exceed $20 \%$ at 1 SLPM anywhere in the thorax model, whereas with 4 SLPM it is already possible to reach $90 \%$ in the "atrium incision." Overall, the CarbonAid device performs better than the other devices when suction is applied, but the difference with other devices is most notable in the "aortic incision." In contrast with the effect of suction, a tilted orientation of the diffusor, has no effect on the $\mathrm{Max} \mathrm{CO}_{2}$ concentration or rise time for

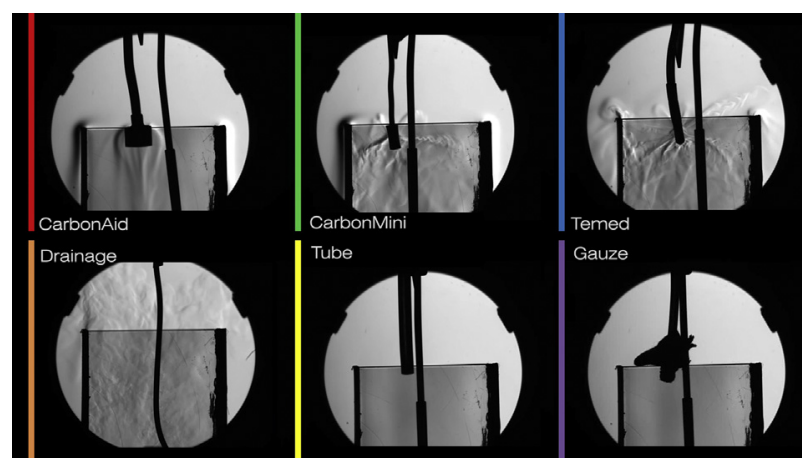

VIDEO 4. All tested diffusors are shown side by side, dispersing $\mathrm{CO}_{2}$ in the rectangular thorax model at a flow rate of 10 SLPM. Video available at: https://www.jtcvs.org/article/S0022-5223(19)30936-5/fulltext. 


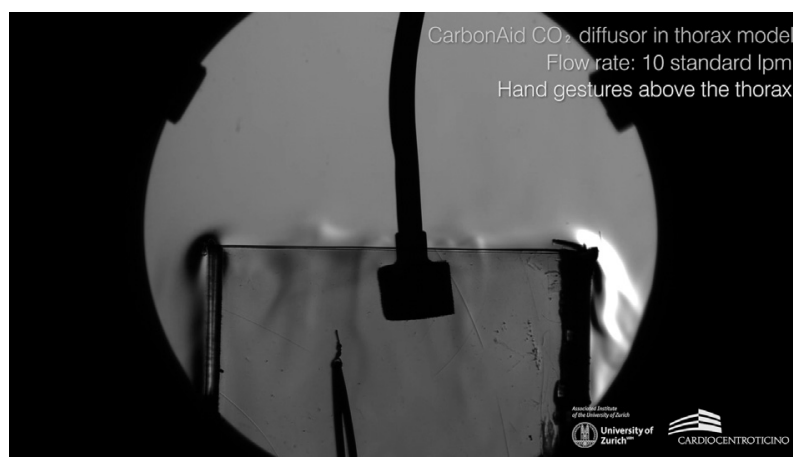

VIDEO 5. Three scenes with a sequence of disturbances are displayed to illustrate the (in)stability of the $\mathrm{CO}_{2}$ atmosphere (maintained by a CarbonAid diffusor at 10 SLPM) and the intermixing of air into the thorax. First, the effect of rapid hand motions above the thorax are shown. Second, knotting and cutting a suture is simulated inside the thorax. Third, the effect introducing a sucker inside the thorax is visualized. Video available at: https://www.jtcvs.org/article/S0022-5223(19)30936-5/fulltext.

either incision region, regardless of the flow rate. For the cases in which $100 \% \mathrm{CO}_{2}$ could be achieved, the rise time with a tilted diffusor was less than 5 seconds longer
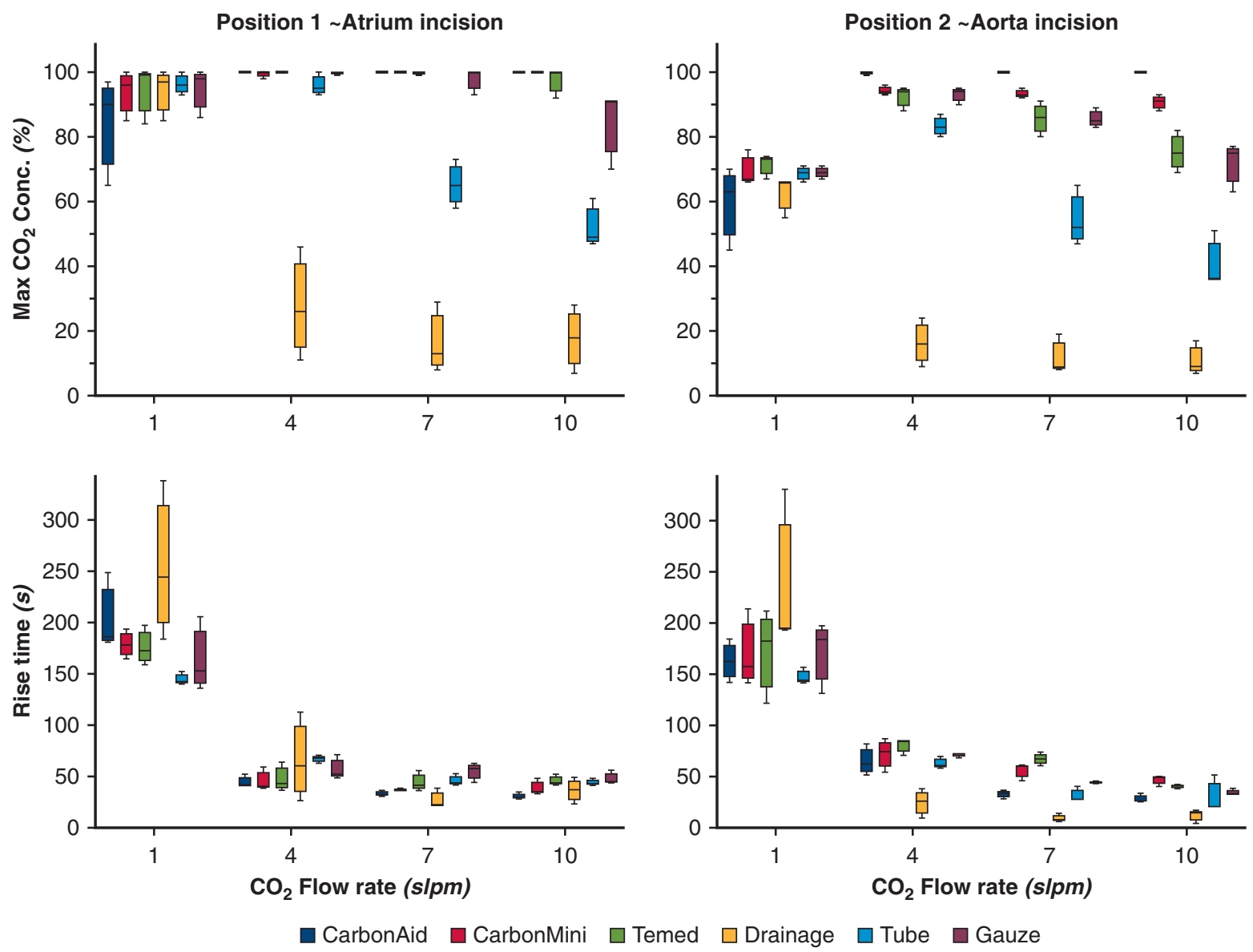

FIGURE 5. Comparison of maximum achieved $\mathrm{CO}_{2}$ concentrations (top) and rise time (bottom) at various $\mathrm{CO}_{2}$ flow rates (1, 4, 7, or $10 \mathrm{SLPM}$ ) for all 6 tested devices. The Tukey box plots are composed of the 3 replications measured for each device-flow rate combination. Left: results at the level of an atrial incision (position 1). Right: results at the level of an aorta incision (position 2). $\mathrm{CO}_{2}$, Carbon dioxide; $S L P M$, standard liter per minute.

than with a vertically placed diffusor, whereas the application of suction would increase the necessary rise time by approximately 10 seconds, indicating that applying suction mostly impacts the maximum achievable gas concentration.

\section{Disturbances}

In addition to the described imaging, we further observed that the $\mathrm{CO}_{2}$ atmosphere is easily disturbed. It appears that mostly air flows cause drastic mixing of air and $\mathrm{CO}_{2}$, while gentle motions like knotting or manipulating small instruments have little effect. We illustrated this in Video 5, in which the disturbances are visible as more intense shadow gradients and nonsteady shadow motions. The last section of Video 5 shows the use of a sucker, in which the rapid removal of $\mathrm{CO}_{2}$ causes turbulent entrainment of air. The 2-dimensional projection may cause the illusion that the sucker is actually blowing air, but it can be observed that introduction of the sucker in the model reduces the overflow of $\mathrm{CO}_{2}$ from the edge of the thorax model, indicating that part of the delivered $\mathrm{CO}_{2}$ volume is evacuated via the sucker. 
TABLE 2. Summary of maximum $\mathrm{CO}_{2}$ concentration differences

\begin{tabular}{|c|c|c|c|c|c|c|c|c|c|c|c|c|c|c|c|c|c|c|c|c|c|}
\hline \multirow[b]{2}{*}{ CarbonAid } & \multirow{2}{*}{ CarbonAid } & \multicolumn{4}{|c|}{ CarbonMini } & \multicolumn{4}{|c|}{ Temed } & \multicolumn{4}{|c|}{ Drainage } & \multicolumn{4}{|c|}{ Tube } & \multicolumn{4}{|c|}{ Gauze } \\
\hline & & -4 & 6 & 7 & 9 & -10 & 6 & 14 & 25 & -3 & 84 & 91 & 91 & -6 & 17 & 48 & 64 & -6 & 6 & 15 & 25 \\
\hline CarbonMini & & & & & & -6 & 0 & 7 & 16 & 1 & 78 & 84 & 82 & -2 & 11 & 41 & 55 & -2 & 0 & 8 & 16 \\
\hline Temed & & & & & & & & & & 7 & 78 & 77 & 66 & 4 & 11 & 34 & 39 & 4 & 0 & 1 & 0 \\
\hline Drainage & & & & & & & & & & & & & & -3 & -67 & -43 & -27 & -3 & -78 & -76 & -66 \\
\hline Tube & & & & & & & & & & & & & & & & & & 0 & -11 & -33 & -39 \\
\hline
\end{tabular}

Pairwise comparison (difference of median) of the max. achieved $\mathrm{CO}_{2}$ concentrations (\%) for 1,4, 7, or 10 SLPM (left to right subcolumns). Positive values (bold) mean left-side device reaches a higher concentration than the top row device, and italic values mean the opposite. Concentrations were measured in position 2 ( aorta incision) with the device positioned vertically, without application of suction (experiment A). Statistical differences in this pairwise comparison could not be assessed because of limitations of the data set.

\section{DISCUSSION}

We set up a simple simulation of the surgical field after sternotomy with $\mathrm{CO}_{2}$ concentration sensors and an imaging technique to visualize the $\mathrm{CO}_{2}$ clouds generated by in situ diffusors to understand the effectiveness of $\mathrm{CO}_{2}$ field flooding and its determining factors.

A first general observation is that the $\mathrm{CO}_{2}$ atmosphere generated by all diffusors is easily disturbed and does not guarantee prevention of blood-air contact. Visualization showed that $\mathrm{CO}_{2}$ behaves in one way much like water: It overflows over the edges of the incision and falls straight down to the floor, while at the same time it is light and fluidic enough to easily generate turbulent flow patterns that enable intermixing and entrainment of air. This observation suggests that $\mathrm{CO}_{2}$ generally does not reach the head level of the surgical staff, although at high flow rates and depending on the diffusor and its orientation, it is possible that $\mathrm{CO}_{2}$ is blown up to this level, as was seen with the use of a drainage catheter at 7 and 10 SLPM (Videos 3 and 4). It also takes time to (re)build a $\mathrm{CO}_{2}$ atmosphere up to its maximum possible concentration at the start of surgery or after disturbing this atmosphere. With approximately 3 minutes of

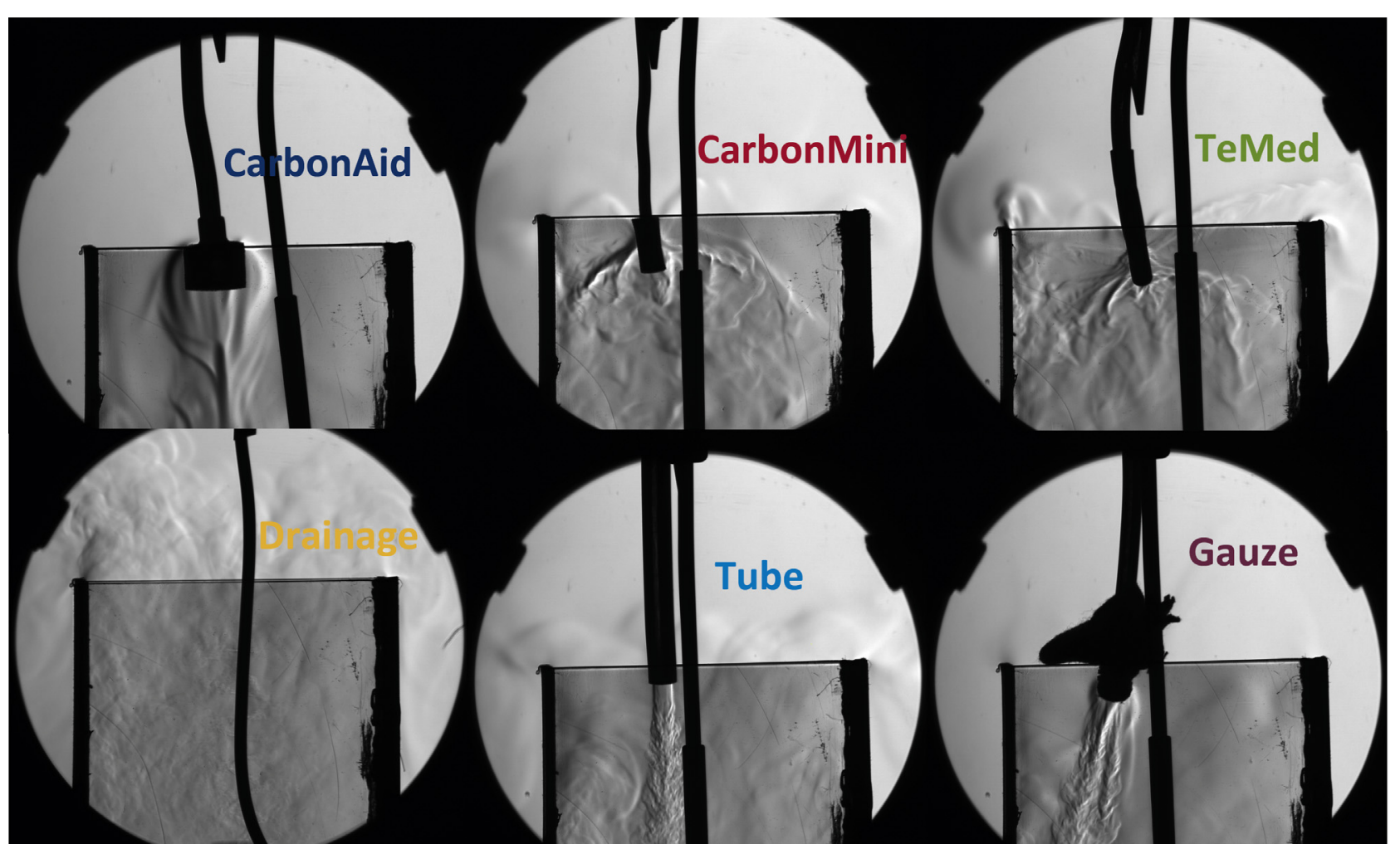

FIGURE 6. Optical comparison of the gas clouds generated by the 6 different vertically placed diffusors at a flow rate of 7 SLPM. In all panels except for the one labeled "drainage," a vertical shadow of the caudally placed suction tip is visible, but this was not actively sucking at the time of recording. This figure is a collage of snap shots of the footage that also composes Video 3. 

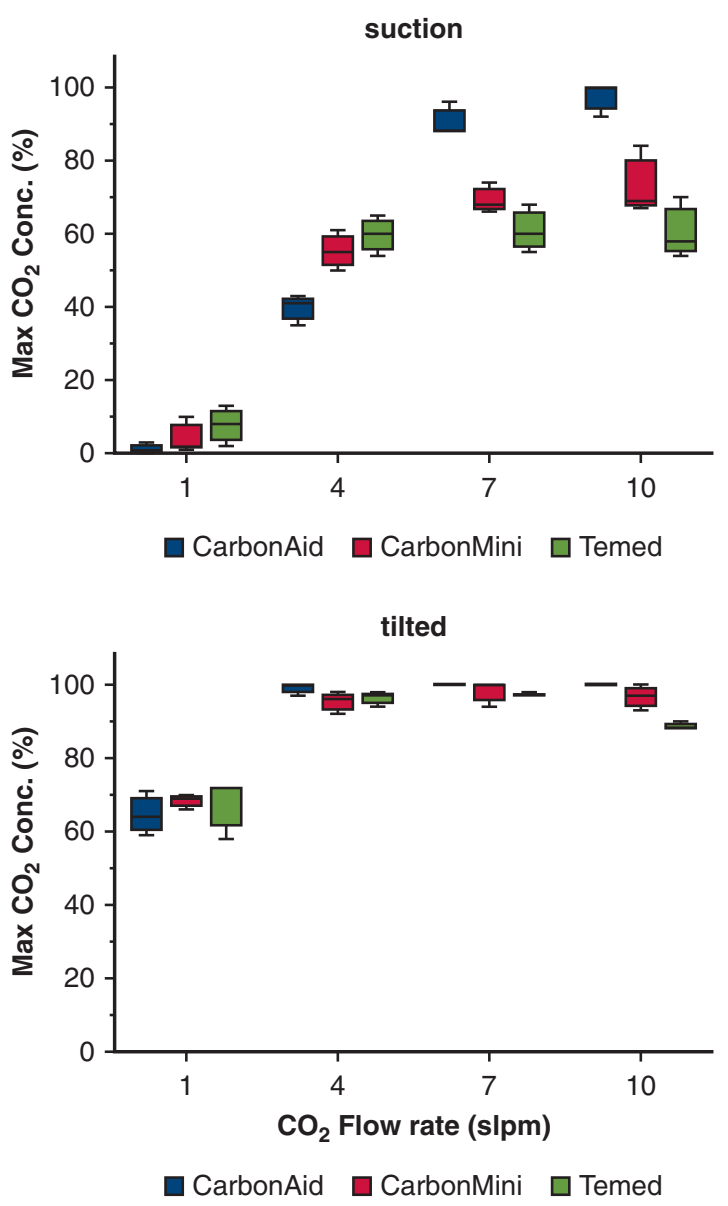

FIGURE 7. Maximum achieved $\mathrm{CO}_{2}$ concentrations in the thorax model at the level of an aorta incision (position 2), generated with 3 different commercial $\mathrm{CO}_{2}$ diffusors at various $\mathrm{CO}_{2}$ flow rates $(1,4,7$, or 10 SLPM). The Tukey box plots are composed of the 3 replications measured for each device-flow rate combination. Top: with the diffusors placed vertically, while a caudally placed blood sucker draws out the gas mixture at 5 SLPM. Bottom: with the diffusors tilted $45^{\circ}$ in the sagittal plane and no active suction. $\mathrm{CO}_{2}$, Carbon dioxide; $S L P M$, standard liter per minute.

rise time at 1 SLPM, we anticipate that this atmosphere will constantly be disturbed during surgery and not have the chance to establish its maximum potential, which is already well below the potential of higher flow rates. The exception is the drainage catheter, which reaches its highest possible concentration at 1 SLPM, but with a median rise time greater than 4 minutes it appears to be impractical and still allows approximately $40 \%$ of air near the aorta.

From our results, it is obvious that the tested drainage catheter performs the worst of all devices and never creates an atmosphere that can be expected to yield any benefits for the prevention of blood-air contact. The video footage clearly reveals extended turbulences and $\mathrm{CO}_{2}$-air mixing, while the concentration measurements confirm this with very low values. We hypothesize that at a low flow rate, the gas velocity coming out of the catheter holes does not entrain much air but needs a long time to fill the thorax, while at higher flow rates, the high velocity jets constantly entrain air and prevent an increase in $\mathrm{CO}_{2}$ concentration. Although we could not prove that position of the diffusor plays a role in the effectiveness of field-flooding, the location of the surgical incision certainly determines the residual risk of blood-air contact, where incisions deeper in the thorax are deemed safer.

In contrast, the CarbonAid diffusor created a $100 \% \mathrm{CO}_{2}$ atmosphere under most circumstances and does this via a mostly laminar flow that falls down gently from this large diffusor. These observations corroborate the findings of Svenarud with gas samplings. ${ }^{20}$ Only with the lowest flow rate (1 SLPM) or with the application of suction, does the CarbonAid max out at lower $\mathrm{CO}_{2}$ concentrations, especially in the region of an aorta incision. The 1 SLPM flow rate in general is unusable for air emboli protection, as explained earlier, because it takes too much time to establish a steady atmosphere and the use of suction would immediately eliminate all the gas. In fact, suction reduces the effectiveness of $\mathrm{CO}_{2}$ field-flooding in 2 ways: On the one hand, it aspirates part of the delivered $\mathrm{CO}_{2}$ gas, while on the other hand it causes disturbances and whirls that entrain air in the thorax. However, we need to point out that although it is obvious that a $100 \% \mathrm{CO}_{2}$ atmosphere is the most certain way to prevent air emboli, it is not disproven that a lower value may suffice as well. Future studies will need to identify a safe cutoff to make an objective device selection.

Gerriets and colleagues ${ }^{21}$ concluded that gaseous microemboli contribute to neuropsychologic decline 3 months postoperatively for coronary artery bypass grafting, indicating that clinical studies also need to distinguish between cardiopulmonary bypass components and blood-air contact as the sources of microemboli to fully understand the effectiveness of $\mathrm{CO}_{2}$ field flooding. Meanwhile, it is a simple, harmless technique that can only yield benefits, especially if some understanding of the gas behavior is present, for which our optical technique is suitable. We have summarized our personal in vitro experience with all tested devices in Table 3.

One covariable we did not assess and that may have an impact on efficiency of $\mathrm{CO}_{2}$ dispersion is the height and locations at which the diffusor is placed in the thorax cavity. However, the geometry of the diffusor plays a role in how and where it can be placed, and our rectangular thorax model is simple compared with the real anatomy.

Besides this simplification, there are other factors present in an operating theatre not considered in this study. The experiments were performed in a laboratory environment without laminar downflow, fans or heat coming of the various devices normally present in the room. This may have a major impact on the air streams in the theatre and on the $\mathrm{CO}_{2}$ atmosphere in and above the thorax and warrants further research. 
TABLE 3. Summary of practical findings

\begin{tabular}{|c|c|c|c|c|}
\hline \multirow[t]{2}{*}{ Devices } & \multicolumn{4}{|c|}{$\mathrm{CO}_{2}$ flow rate (SLPM) } \\
\hline & 1 & 4 & 7 & 10 \\
\hline CarbonAid & \multirow{6}{*}{$\begin{array}{l}\text { Rise time } \\
\text { long. Very } \\
\text { sensitive to } \\
\text { suction, and } \\
\text { disturbances }\end{array}$} & \multirow{5}{*}{$\begin{array}{l}\text { Highly ineffective } \\
\text { with suction or air } \\
\text { flow disturbances; } \\
\text { acceptable if these } \\
\text { are avoided }\end{array}$} & Ideal combination & $\begin{array}{c}\text { Effective, but } \\
\text { excessive flow rate }\end{array}$ \\
\hline CarbonMini & & & \multirow{3}{*}{$\begin{array}{c}\text { Acceptable } \\
\text { without suction }\end{array}$} & $\begin{array}{c}\text { Acceptable without } \\
\text { suction }\end{array}$ \\
\hline Temed & & & & \multirow{2}{*}{$<7$ SLPM } \\
\hline Gauze & & & & \\
\hline Tube & & & \multicolumn{2}{|c|}{ Higher flow rate is less effective } \\
\hline Drainage & & \multicolumn{3}{|c|}{ Insufficient $\mathrm{CO}_{2}$ concentration } \\
\hline Color Legend & Best option & Acceptable & $\begin{array}{c}\text { Not } \\
\text { recommended }\end{array}$ & Worst option \\
\hline
\end{tabular}

Subjective evaluation of the effectiveness of the different devices used at various flow rates (SLPM) in the rectangular thorax model. The evaluation was based on the authors' interpretation of the rise time and maximum concentration results, the video imaging und unreported tests. $\mathrm{CO}_{2}$, Carbon dioxide; $S L P M$, standard liter per minute.

\section{CONCLUSIONS}

Our visualization and concentration measurements agreed well and indicate that the CarbonAid device is superior to the other tested diffusors, where improvised devices are clearly inferior to commercial ones.

The flow rate of $\mathrm{CO}_{2}$ delivery is an important factor, and higher is not always better, but a low flow rate like 1 SLPM has no merits for avoiding blood-air contact in an open thorax. The $\mathrm{CO}_{2}$ atmosphere generated by any diffusor is unstable, and there are numerous factors that can disturb it, where suction in the surgical field is certainly a key issue.

\section{Conflict of Interest Statement}

Authors have nothing to disclose with regard to commercial support.

This study was made possible in part with the financial support of the Commission for Technology and Innovation of the Swiss
Government (CTI-19212.1). The authors are grateful for the assistance of Franco Dossena of the Cardiocentro Ticino for the professional video editing and to Catherine Klersy of the Policlinico San Matteo for the statistical analysis.

\section{References}

1. Chung EML, Banahan C, Patel N, Janus J, Marshall D, Horsfield MA, et al. Size distribution of air bubbles entering the brain during cardiac surgery. PLoS One. 2015;10:1-11.

2. Hindman BJ, Dexter F, Subieta A, Smith T, Cutkomp J. Brain injury after cerebral arterial air embolism in the rabbit as determined by triphenyltetrazolium staining. Anesthesiology. 1999;90:1462-73.

3. Lamy A. NeuroVISION Cardiac Pilot CCS. Presented at the 32nd EACTS annual meeting, Milan; 2018. Available at: http://medialibrary.eacts.cyim.com/media theque/media.aspx ?mediaId $=51976 \&$ channel $=10233$. Accessed March 10, 2019.

4. Kopjar T. Silent brain injury associated to postoperative cognitive decline after coronary bypass surgery. Presented at the 32 nd EACTS annual meeting, Milan; 2018. Available at: http://medialibrary.eacts.cyim.com/mediatheque/media aspx?mediaId=51977\&channel=10233. Accessed March 10, 2019.

5. Floyd TF, Shah PN, Price CC, Harris F, Ratcliffe SJ, Acker MA, et al. Clinically silent cerebral ischemic events after cardiac surgery: their incidence, regional 
vascular occurrence, and procedural dependence. Ann Thorac Surg. 2006;81: 2160-6.

6. Borger MA, Peniston CM, Weisel RD, Vasiliou M, Green RE, Feindel CM. Neuropsychologic impairment after coronary bypass surgery: effect of gaseous microemboli during perfusionist interventions. J Thorac Cardiovasc Surg. 2001; 121:743-9.

7. Taylor RL, Borger MA, Weisel RD, Fedorko L, Feindel CM. Cerebral microemboli during cardiopulmonary bypass: increased emboli during perfusionist interventions. Ann Thorac Surg. 1999;68:89-93.

8. Myerly WH, Throckmorton TD, Gustafson JE. The closure of cardiac septal defects; the beginning of by-pass surgery at a medium-sized private hospital. AMA Arch Surg. 1957;74:918-26.

9. Giordano S, Biancari F. Does the use of carbon dioxide field flooding during heart valve surgery prevent postoperative cerebrovascular complications? Interact Cardiovasc Thorac Surg. 2009;9:323-6.

10. Sun Y, Ji B, Zhu X, Zheng Z. Efficacy of carbon dioxide insufflation for cerebral and cardiac protection during open heart surgery: a systematic review and metaanalysis. Artif Organs. 2013;37:439-46.

11. Chaudhuri K, Storey E, Lee GA, Bailey M, Chan J, Rosenfeldt FL, et al. Carbon dioxide insufflation in open-chamber cardiac surgery: a double-blind, randomized clinical trial of neurocognitive effects. J Thorac Cardiovasc Surg. 2012; 144:646-53.e1.

12. Chatterjee S, Greenberg SB, Brown J, Murphy GS, Pearson PJ, Alexander JC. Simple technique to verify $\mathrm{CO} 2$ diffusion with the CarbonAid device. Heart Surg Forum. 2012;15:212.

13. Frey JMK, Janson M, Svanfeldt M, Svenarud PK, Van Der Linden JA. Intraoperative local insufflation of warmed humidified $\mathrm{CO} 2$ increases open wound and core temperatures: a randomized clinical trial. World J Surg. 2012;36: 2567-75.
14. Svenarud P, Persson M, Van der Linden J. Efficiency of a gas diffuser and influence of suction in carbon dioxide deairing of a cardiothoracic wound cavity model. J Thorac Cardiovasc Surg. 2003;125:1043-9.

15. Martens S, Neumann K, Sodemann C, Deschka H, Wimmer-Greinecker G, Moritz A. Carbon dioxide field flooding reduces neurologic impairment after open heart surgery. Ann Thorac Surg. 2008;85:543-7.

16. Skidmore KL, Jones C, DeWet C. Flooding the surgical field with carbon dioxide during open heart surgery improves segmental wall motion. J Extra Corpor Technol. 2006;38:123-7.

17. Al-Rashidi F, Landenhed M, Blomquist S, Höglund P, Karlsson PA, Pierre L, et al. Comparison of the effectiveness and safety of a new de-airing technique with a standardized carbon dioxide insufflation technique in open left heart surgery: a randomized clinical trial. J Thorac Cardiovasc Surg. 2011;141: 1128-33.

18. Landenhed M, Al-Rashidi F, Blomquist S, Höglund P, Pierre L, Koul B. Systemic effects of carbon dioxide insufflation technique for de-airing in left-sided cardiac surgery. J Thorac Cardiovasc Surg. 2014;147:295-300.

19. Pugsley W, Klinger L, Paschalis C, Treasure T, Harrison M, Newman S. The impact of microemboli during cardiopulmonary bypass on neuropsychological functioning. Stroke. 1994;25:1393-9.

20. Svenarud PK. Carbon dioxide de-airing in cardiac surgery [doctoral thesis]. Stockholm, Sweden: Karolinska University; 2004.

21. Gerriets T, Schwarz N, Sammer G, Baehr J, Stolz E, Kaps M, et al. Protecting the brain from gaseous and solid micro-emboli during coronary artery bypass grafting: a randomized controlled trial. Eur Heart J. 2009;31:360-8.

Key Words: carbon dioxide, field-flooding, air emboli, prevention, neuroprotection 